\title{
Bounded and unbounded non-oscillatory solutions of a four-dimensional nonlinear neutral difference system
}

\author{
Josef Diblík , Barbara Łupińska², Miroslava Růžičková ${ }^{3 *}$ and Joanna Zonenberg²
}

"Correspondence:

miroslava.ruzickova@fpv.uniza.sk ${ }^{3}$ University of Žilina, Žilina, Slovakia

Full list of author information is available at the end of the article

\begin{abstract}
The paper is concerned with a four-dimensional nonlinear difference system with delayed arguments where the first equation of the system is of a neutral type. A classification of non-oscillatory solutions is given and results on their boundedness or unboundedness are derived. The results obtained are illustrated by examples.
\end{abstract}

MSC: 39A10; 39A11; 39A12

Keywords: difference equation; neutral type equation; nonlinear system; non-oscillatory solution; bounded solution; unbounded solution

\section{Introduction}

In the paper, we consider a nonlinear four-dimensional neutral difference system of equations being a particular case of a general system of the form

$$
\Delta(X(n)+P(n) X(n-\delta))=A(n) F(X(n-\tau)), \quad n \geq n_{0},
$$

where $n_{0} \in \mathbb{N}, \delta$, and $\tau$ are nonnegative integers,

$$
X(n)=\left(x_{1}(n), x_{2}(n), x_{3}(n), x_{4}(n)\right)^{T}: \mathbb{Z}_{n_{0}-\eta}^{\infty} \rightarrow \mathbb{R}^{4}
$$

is an unknown vector, $\mathbb{Z}_{n_{0}-\eta}^{\infty}:=\left\{n_{0}-\eta, n_{0}-\eta+1, \ldots\right\}, \eta=\max \{\delta, \tau\}, \Delta$ is the forward difference operator, $\Delta X(n)=X(n+1)-X(n)$,

$$
P(n), A(n): \mathbb{Z}_{n_{0}}^{\infty} \rightarrow \mathbb{R}^{4} \times \mathbb{R}^{4}
$$

are squared matrices and $F(n): \mathbb{Z}_{n_{0}-\eta}^{\infty} \rightarrow \mathbb{R}^{4}$. The particular case is specified in the following way:

$$
\begin{aligned}
& P(n):=\operatorname{diag}(p(n), 0,0,0), \quad A(n):=\operatorname{diag}\left(a_{1}(n), a_{2}(n), a_{3}(n), a_{4}(n)\right), \\
& F(n):=\left(f_{1}\left(x_{2}(n)\right), f_{2}\left(x_{3}(n)\right), f_{3}\left(x_{4}(n)\right), f_{4}\left(x_{1}(n)\right)\right)^{T}
\end{aligned}
$$

(c) 2015 Diblík et al. This article is distributed under the terms of the Creative Commons Attribution 4.0 International License (http://creativecommons.org/licenses/by/4.0/), which permits unrestricted use, distribution, and reproduction in any medium, provided you give appropriate credit to the original author(s) and the source, provide a link to the Creative Commons license, and indicate if changes were made. 
and $f_{i}: \mathbb{R} \rightarrow \mathbb{R}, i=1, \ldots, 4$. Throughout the paper, we assume that the sequence $\{p(n)\}$ satisfies

$$
\lim _{n \rightarrow \infty} p(n)=\bar{p} \in \mathbb{R} \quad \text { and } \quad|\bar{p}| \neq 1 .
$$

When formulating and proving statements, we understand that (2) is valid without mentioning this property.

Obviously, system (1) can be written as

$$
\begin{aligned}
& \Delta\left(x_{1}(n)+p(n) x_{1}(n-\delta)\right)=a_{1}(n) f_{1}\left(x_{2}(n-\tau)\right), \\
& \Delta x_{2}(n)=a_{2}(n) f_{2}\left(x_{3}(n-\tau)\right), \\
& \Delta x_{3}(n)=a_{3}(n) f_{3}\left(x_{4}(n-\tau)\right), \\
& \Delta x_{4}(n)=a_{4}(n) f_{4}\left(x_{1}(n-\tau)\right) .
\end{aligned}
$$

Throughout the paper, we assume

$$
\begin{aligned}
& a_{i}: \mathbb{Z}_{n_{0}}^{\infty} \rightarrow \mathbb{R}_{+} \cup\{0\}, \quad \mathbb{R}_{+}:=(0, \infty), \sum_{n=1}^{\infty} a_{i}(n)=\infty, \quad i=1,2,3, \\
& a_{4}: \mathbb{Z}_{n_{0}}^{\infty} \rightarrow \mathbb{R}^{4} \rightarrow \mathbb{R}_{+} .
\end{aligned}
$$

Moreover, let a constant $M>0$ exist such that

$$
\frac{f_{i}(u)}{u} \geq M, \quad u \in \mathbb{R} \backslash\{0\}, i=1, \ldots, 4 .
$$

This property implies $u f_{i}(u)>0$ for $u \neq 0, i=1, \ldots, 4$.

The properties of solutions to second-order difference equations (such as oscillatory or non-oscillatory behavior) have been the subject of intensive studies in the last 20 years.

Considerably less attention received the study of similar properties to special classes of fourth-order nonlinear difference equations. Some interesting results concerning asymptotic and oscillatory properties of the fourth-order nonlinear difference equations can be find in $[1,2]$. A systematic investigation of the behavior properties of solutions of some classes of fourth-order nonlinear difference equations can be found in [3]. The authors presented sufficient conditions for the oscillation of solutions via comparison with the first and second-order difference equations whose oscillatory behavior is well known. The study of various kinds of fourth-order nonlinear difference equations are brought to the attention of many authors (see, e.g., [4-19] and the references therein).

A four-dimensional system of difference equations, which can be understood as a generalization of fourth-order difference equations, was investigated, e.g., in [20-22]. The boundedness and oscillation of nonlinear three-dimensional difference systems with delays were considered in [23, 24].

Provided that functions $f_{i}$ are invertible and $a_{i}(n) \neq 0$ for $i=1,2,3, n \in \mathbb{Z}_{n_{0}-\eta}^{\infty}$, system (1) can be rewritten as a fourth-order difference equation,

$$
\begin{aligned}
& \Delta\left(f_{3}^{-1}\left(b_{3}(n) \Delta\left(f_{2}^{-1}\left(b_{2}(n) \Delta\left(f_{1}^{-1}\left(b_{1}(n) \Delta\left(x_{1}(n)+p(n) x_{1}(n-\delta)\right)\right)\right)\right)\right)\right)\right) \\
& \quad=b_{4}(n) f_{4}\left(x_{1}(n-4 \tau)\right), \quad n \in \mathbb{Z}_{n_{0}}^{\infty},
\end{aligned}
$$


where $b_{1}(n)=1 / a_{1}(n), b_{2}(n)=1 / a_{2}(n-\tau), b_{3}(n)=1 / a_{3}(n-2 \tau)$, and $b_{4}(n)=1 / a_{4}(n-3 \tau)$. The special case of (10) with power-type functions $f_{i}, i=1, \ldots, 4$ was considered in [1] where the authors, among others, stated a new oscillation theorem for what is called the sublinear case. Their approach was based on regarding the equation as a four-dimensional difference system and on the cyclic permutation of the coefficients in the difference equations. The equation

$$
\Delta\left(b_{3}(n) \Delta\left(b_{2}(n) \Delta\left(b_{1}(n) \Delta\left(x_{1}(n)+p(n) x_{1}(n-\delta)\right)\right)\right)\right)=b_{4}(n) f\left(x_{1}(n)\right)
$$

is considered in [25] as a special case of (10) where $f_{i}(t)=t, i=1,2,3$, and $f_{4}\left(x_{1}(n-4 \tau)\right)=$ $f\left(x_{1}(n)\right)$. Conditions under which every eventually positive solution belongs to one of three mutually distinct sets are derived. Also, necessary and sufficient conditions for the existence of asymptotically constant solutions and maximal solutions are given. The properties of non-oscillatory solutions of (11) are investigated in [26] where the necessary and sufficient conditions for the existence of minimal and maximal solutions are given as well.

By a solution of (1) we mean a vector $X=X(n), n \in \mathbb{Z}_{n_{0}-\eta}^{\infty}$ such that, for every $n \in \mathbb{Z}_{n_{0}}^{\infty}$, (1) is fulfilled. A solution $X=X(n), n \in \mathbb{Z}_{n_{0}-\eta}^{\infty}$ of (1) is said to be non-oscillatory if each of its components is non-oscillatory (i.e. it is always positive or is always negative) on $\mathbb{Z}_{n_{0}-\eta}^{\infty}$. A solution $X=X(n), n \in \mathbb{Z}_{n_{0}-\eta}^{\infty}$ of (1) is said to be eventually non-oscillatory if all its components are non-oscillatory for all sufficiently large $n$. Otherwise (if a solution is neither non-oscillatory nor eventually non-oscillatory), it is called oscillatory. A solution $X=X(n)$, $n \in \mathbb{Z}_{n_{0}-\eta}^{\infty}$ of (1) is said to be bounded if all its components are bounded, otherwise, it is called unbounded. In the paper, we often assume or state that a property holds 'eventually' meaning that such property is valid for all sufficiently large values of the independent variable $n$. The rest of the paper is organized as follows. In Section 2, some auxiliary results are introduced. The main results are proved in Section 3 and, in the last Section 4, we illustrate selected results by examples.

\section{Auxiliary results}

For convenience, in our investigation we denote

$$
z(n)=x_{1}(n)+p(n) x_{1}(n-\delta), \quad n \in \mathbb{Z}_{n_{0}}^{\infty}
$$

We will employ some auxiliary results to prove the main results of the paper. First, recall two lemmas, which can be found in [27] and in [28], respectively.

Lemma 1 Let $\left\{x_{1}(n)\right\}, n \in \mathbb{Z}_{n_{0}-\eta}^{\infty},\{p(n)\}, n \in \mathbb{Z}_{n_{0}}^{\infty}$, be real sequences and $\{z(n)\}$ be the sequence defined by (12). If $\left\{x_{1}(n)\right\}$ is bounded, and

$$
\lim _{n \rightarrow \infty} z(n)=l \in \mathbb{R}
$$

then $\left\{x_{1}(n)\right\}$ is convergent and

$$
\lim _{n \rightarrow \infty} x_{1}(n)=\frac{l}{1+\bar{p}} .
$$

Remark 1 If conditions of Lemma 1 hold when $l \neq 0$, then the sequences $\left\{x_{1}(n)\right\}$ and $\{z(n)\}$ are both eventually non-oscillatory. 
Lemma 2 Let $\left\{x_{1}(n)\right\}, n \in \mathbb{Z}_{n_{0}-\eta}^{\infty},\{p(n)\}, n \in \mathbb{Z}_{n_{0}}^{\infty}$ be real sequences and $\{z(n)\}$ be a sequence defined by (12). Assume also that

$$
|\bar{p}|<1
$$

If the sequence $\{z(n)\}$ defined by (12) is bounded, then the sequence $\left\{x_{1}(n)\right\}$ is bounded as well.

Remark 2 If conditions of Lemma 2 hold, then the sequence $\{z(n)\}$ is bounded if and only if the sequence $\left\{x_{1}(n)\right\}$ is bounded. Thus, the sequence $\{z(n)\}$ is unbounded if and only if the sequence $\left\{x_{1}(n)\right\}$ is unbounded.

Next, we will prove some properties of the solutions concerning sign, monotonicity and convergence to zero, formulating them as lemmas.

Lemma 3 If conditions (13) and (14) are satisfied and $l \neq 0$, then the sequences $\left\{x_{1}(n)\right\}$ and $\{z(n)\}$ have the same sign for all sufficiently large $n$.

Proof Since the sequence $\{z(n)\}$ has a finite limit, it is bounded. By Lemma 2 , the sequence $\left\{x_{1}(n)\right\}$ is also bounded. By Lemma 1, using the usual rules for computing limits, we have

$$
\lim _{n \rightarrow \infty} x_{1}(n)=\frac{1}{1+\bar{p}} \cdot \lim _{n \rightarrow \infty} z(n)
$$

Since $|\bar{p}|<1$, both $x_{1}(n)$ and $z(n)$ have the same sign if $n$ is sufficiently large. This implies the lemma's assertion.

The following lemma can be immediately proved if, in the system (1) written by (3)-(6), single equations are analyzed separately (starting with (6)).

Lemma 4 Let $X=X(n), n \in \mathbb{Z}_{n_{0}-\eta}^{\infty}$ be a solution of the system (1) such that the sequence $\left\{x_{1}(n)\right\}$ is non-oscillatory. If conditions (7)-(9) are satisfied, $X$ is eventually non-oscillatory and the sequences $\left\{x_{i}(n)\right\}, i=2,3,4$, are monotonic for all sufficiently large $n$. Moreover, the sequence $\{z(n)\}$ defined by $(12)$ is also monotonic for all sufficiently large $n$.

Lemma 5 Let $X=X(n), n \in \mathbb{Z}_{n_{0}-\eta}^{\infty}$ be a solution of the system (1), and conditions (7)-(9) be satisfied. If $\left\{x_{1}(n)\right\}$ is non-oscillatory and $\lim _{n \rightarrow \infty} x_{1}(n)$ is finite, then

$$
\lim _{n \rightarrow \infty} x_{i}(n)=0, \quad i=2,3,4
$$

Proof Set $\lim _{n \rightarrow \infty} x_{1}(n)=c_{1}$. Hence, $\lim _{n \rightarrow \infty} z(n)=c_{1}(1+\bar{p})$ is finite, too. By Lemma 4 , the sequence $\left\{x_{2}(n)\right\}$ is monotonic. Hence, there exists a limit

$$
\lim _{n \rightarrow \infty} x_{2}(n)=c_{2} \in \mathbb{R} \cup\{-\infty,+\infty\}
$$

Without loss of generality, assume that $c_{2} \geq 0$. If $c_{2}=0$, then the statement of the lemma is valid for $i=2$. Inequality $c_{2}>0$ yields $x_{2}(n)>0$ for all sufficiently large $n$. Then there exists 
an integer $n_{1} \in \mathbb{Z}_{n_{0}-\eta}^{\infty}$ such that $x_{2}(n-\tau) \geq c_{2} / 2$ for all $n \geq n_{1}+\tau$. From (9), we derive $f_{1}\left(x_{2}(n-\tau)\right) \geq M x_{2}(n-\tau)>0$. Thus, from (3), we have

$$
\Delta z(n)=a_{1}(n) f_{1}\left(x_{2}(n-\tau)\right) \geq M a_{1}(n) x_{2}(n-\tau) \geq M a_{1}(n) \frac{c_{2}}{2}>0
$$

for $n \geq n_{1}+\tau$. Summing the above inequality from $n_{1}+\tau$ to $n-1$, we get

$$
z(n) \geq z\left(n_{1}+\tau\right)+M \frac{c_{2}}{2} \sum_{i=n_{1}+\tau}^{n-1} a_{1}(i)
$$

Taking $n \rightarrow \infty$, by (7), the right-hand side of the above inequality tends to infinity. So, the left-hand side also tends to infinity. This contradicts the fact that $\lim _{n \rightarrow \infty} z(n)$ is finite. Therefore, $\lim _{n \rightarrow \infty} x_{2}(n)=0$. In a similar way, using the equations of system (1), we obtain $\lim _{n \rightarrow \infty} x_{3}(n)=0$ and $\lim _{n \rightarrow \infty} x_{4}(n)=0$.

\section{Main results}

We focus on the study of boundedness and unboundedness of non-oscillatory solutions of system (1). Before we state sufficient conditions for the solutions to be bounded or unbounded, we give a classification of non-oscillatory solutions of system (1). Investigation of properties of solutions to difference equations and systems relates to the signs of their solutions. Many authors use, e.g., auxiliary results based on the well-known Kiguradze theorem, useful in the theory of functional differential equations. Although our approach is different, it is also based on the signs of solutions.

\subsection{Classification of non-oscillatory solutions}

In the following theorem, we give all possibilities for the signs of components of every eventually non-oscillatory solution $X=X(n), n \in \mathbb{Z}_{n_{0}-\eta}^{\infty}$ of the system (1). Define the following cases related to the eventually non-oscillatory solution $X=X(n), n \in \mathbb{Z}_{n_{0}-\eta}^{\infty}$ of the system (1):

(I) $\operatorname{sgn} x_{1}(n)=\operatorname{sgn} x_{2}(n)=\operatorname{sgn} x_{3}(n)=\operatorname{sgn} x_{4}(n)$,

(II) $\operatorname{sgn} x_{1}(n)=\operatorname{sgn} x_{2}(n)=\operatorname{sgn} x_{3}(n) \neq \operatorname{sgn} x_{4}(n)$,

(III) $\operatorname{sgn} x_{1}(n)=\operatorname{sgn} x_{3}(n) \neq \operatorname{sgn} x_{2}(n)=\operatorname{sgn} x_{4}(n)$

for all sufficiently large $n$.

Theorem 1 Let conditions (7)-(9) and (14) be satisfied. Then the coordinates of every eventually non-oscillatory solution $X=X(n), n \in \mathbb{Z}_{n_{0}-\eta}^{\infty}$ of the system (1) satisfy exactly one of the cases (I)-(III) for all sufficiently large $n$.

Proof Let $X=X(n), n \in \mathbb{Z}_{n_{0}-\eta}^{\infty}$ be an eventually non-oscillatory solution of system (1). Without loss of generality, assume that $x_{1}(n)>0$ for all sufficiently large $n$. There are eight possible cases of the signs of components of $X$ :

(1) $\quad x_{2}(n)>0, \quad x_{3}(n)>0, \quad x_{4}(n)>0$,

(2) $\quad x_{2}(n)>0, \quad x_{3}(n)>0, \quad x_{4}(n)<0$, 
(3) $\quad x_{2}(n)>0, \quad x_{3}(n)<0, \quad x_{4}(n)>0$,

(4) $\quad x_{2}(n)>0, \quad x_{3}(n)<0, \quad x_{4}(n)<0$,

(5) $\quad x_{2}(n)<0, \quad x_{3}(n)>0, \quad x_{4}(n)>0$,

(6) $\quad x_{2}(n)<0, \quad x_{3}(n)>0, \quad x_{4}(n)<0$,

(7) $\quad x_{2}(n)<0, \quad x_{3}(n)<0, \quad x_{4}(n)>0$,

(8) $\quad x_{2}(n)<0, \quad x_{3}(n)<0, \quad x_{4}(n)<0$

for all sufficiently large $n$.

First, we exclude cases (3) and (7). For the sake of contradiction, suppose that

$$
x_{3}(n)<0 \text { and } x_{4}(n)>0
$$

for all sufficiently large $n$. Hence, the sequence $\left\{x_{4}(n)\right\}$ is eventually positive and increasing (by (6)). Then there exists $n_{2} \in \mathbb{Z}_{n_{0}-\eta}^{\infty}$ such that $x_{4}(n-\tau) \geq x_{4}\left(n_{2}\right)>0$ for all $n \geq n_{2}+\tau$. From (5), we obtain

$$
\Delta x_{3}(n) \geq M a_{3}(n) x_{4}(n-\tau) \geq M a_{3}(n) x_{4}\left(n_{2}\right) \quad \text { for } n \geq n_{2}+\tau
$$

Summing up the above inequalities from $n_{2}+\tau$ to $n-1$, we have

$$
x_{3}(n)-x_{3}\left(n_{2}+\tau\right) \geq M x_{4}\left(n_{2}\right) \sum_{i=n_{2}+\tau}^{n-1} a_{3}(i) .
$$

Letting $n$ tend to infinity, the right-hand side of the above inequality tends to $+\infty$. Thus, the left-hand side does so, too. Hence, $\lim _{n \rightarrow \infty} x_{3}(n)=+\infty$. This means that the sequence $\left\{x_{3}(n)\right\}$ is eventually positive. This contradiction excludes both cases (3) and (7).

Case (4). Assume that

$$
x_{2}(n)>0, \quad x_{3}(n)<0 \quad \text { and } \quad x_{4}(n)<0
$$

for all sufficiently large $n$. Then the sequence $\left\{x_{3}(n)\right\}$ is eventually negative and nonincreasing (by (5)), and there exists $n_{3} \in \mathbb{Z}_{n_{0}-\eta}^{\infty}$ such that $x_{3}(n-\tau) \leq x_{3}\left(n_{3}\right)$ for all $n \geq n_{3}+\tau$. From (4), we get

$$
\Delta x_{2}(n) \leq M a_{2}(n) x_{3}(n-\tau) \leq M a_{2}(n) x_{3}\left(n_{3}\right) \text { for } n \geq n_{3}+\tau
$$

Summing up the above inequalities from $n_{3}+\tau$ to $n-1$, we have

$$
x_{2}(n)-x_{2}\left(n_{3}+\tau\right) \leq M x_{3}\left(n_{3}\right) \sum_{i=n_{3}+\tau}^{n-1} a_{2}(i) .
$$

Letting $n$ tend to infinity, the right-hand side of the above inequality tends to $-\infty$. Hence, $\lim _{n \rightarrow \infty} x_{2}(n)=-\infty$. This means that the sequence $\left\{x_{2}(n)\right\}$ is eventually negative. This contradiction excludes the considered case. 
Cases (5) and (8). Suppose that $x_{2}(n)<0$ for all sufficiently large $n$. Hence, using (3), we can prove that the sequence $\{z(n)\}$ is eventually nonincreasing. Since $\{z(n)\}$ is eventually positive, there exists a finite limit of this sequence. By Lemma 2, the sequence $\left\{x_{1}(n)\right\}$ is bounded and, by Lemma $1,\left\{x_{1}(n)\right\}$ has a finite limit. Thus, by Lemma $5, \lim _{n \rightarrow \infty} x_{2}(n)=0$. We conclude that the sequence $\left\{x_{2}(n)\right\}$ is nondecreasing. By $(4)$, the sequence $\left\{x_{3}(n)\right\}$ is eventually positive. This statement excludes the case (8).

Finally, we show that case (5) is not possible either. Suppose that it holds. Then $x_{4}(n)$ is eventually positive and, by (5), the sequence $\left\{x_{3}(n)\right\}$ is eventually nondecreasing, i.e., $\Delta x_{3}(n) \geq 0$ eventually.

By Lemma $5, \lim _{n \rightarrow \infty} x_{3}(n)=0$. Since the sequence $\left\{x_{3}(n)\right\}$ is assumed to be eventually positive, it implies $\Delta x_{3}\left(n_{k}^{*}\right)<0, k=1,2, \ldots$, for a sequence of indices $n_{0}<n_{1}^{*}<n_{2}^{*}<\cdots$ such that $\lim _{k \rightarrow \infty} n_{k}^{*}=\infty$. This contradicts not only the above derived inequality $\Delta x_{3}(n) \geq 0$ for all sufficiently large $n$ but, in the end, case (5), too.

Remark 3 As shown by Examples 1-3 below, the sets of solutions satisfying conditions (I)-(III) of Theorem 1 are nonempty.

\subsection{Bounded and unbounded solutions}

In this part, we give sufficient conditions for the boundedness or unboundedness of nonoscillatory solutions of system (1).

Theorem 2 Assume that conditions (7)-(9) and (14) are satisfied. Let $X=X(n), n \in \mathbb{Z}_{n_{0}-\eta}^{\infty}$ be an eventually non-oscillatory solution of system (1). If

$$
\lim _{n \rightarrow \infty} x_{i}(n) \neq 0 \quad \text { for some } i \in\{2,3,4\}
$$

then $X$ is unbounded.

Proof Since $X=X(n), n \in \mathbb{Z}_{n_{0}-\eta}^{\infty}$ is an eventually non-oscillatory solution of (1), by Lemma 4 , the sequences $\left\{x_{2}(n)\right\},\left\{x_{3}(n)\right\}$, and $\left\{x_{4}(n)\right\}$ are eventually monotonic. Then there exist limits

$$
\lim _{n \rightarrow \infty} x_{i}(n)=c_{i} \neq 0, \quad c_{i} \in \mathbb{R} \cup\{-\infty,+\infty\}, i=2,3,4 .
$$

First, we take $i=2$. Without loss of generality, assume that $c_{2}>0$. Hence, the sequence $\left\{x_{2}(n)\right\}$ is eventually positive. Then there exists an index $n_{4}$ such that $x_{2}(n-\tau)>c_{2} / 2$ for $n \geq n_{4}+\tau$. Using (3), we obtain

$$
\Delta z(n)=a_{1}(n) f_{1}\left(x_{2}(n-\tau)\right) \geq M a_{1}(n) x_{2}(n-\tau) \geq \frac{M c_{2}}{2} a_{1}(n)
$$

for every $n \geq n_{4}+\tau$. Summing up the above inequalities from $n_{4}+\tau$ to $n-1$, we have

$$
z(n) \geq z\left(n_{4}+\tau\right)+M \frac{c_{2}}{2} \sum_{j=n_{4}+\tau}^{n-1} a_{1}(j) .
$$

Letting $n \rightarrow \infty$, by (7), we obtain $\lim _{n \rightarrow \infty} z(n)=\infty$. Then, by Remark 2 , we obtain $\lim _{n \rightarrow \infty} x_{1}(n)=\infty$. This means that $X$ is an unbounded solution of system (1). 
If we take $i=3$ or $i=4$, as above, we get $\lim _{n \rightarrow \infty} x_{2}(n)=0$ or $\lim _{n \rightarrow \infty} x_{3}(n)=0$, respectively.

Theorem 3 If conditions (7)-(9) and (14) are satisfied, then every eventually non-oscillatory solution $X=X(n), n \in \mathbb{Z}_{n_{0}-\eta}^{\infty}$ of (1) fulfilling one of conditions (I), (II) is unbounded.

Proof Let $X=X(n), n \in \mathbb{Z}_{n_{0}-\eta}^{\infty}$ be an eventually non-oscillatory solution of (1). Without loss of generality, assume that the sequence $\left\{x_{1}(n)\right\}$ is eventually positive.

If (I) or (II) hold, then $x_{2}(n)>0$ and $x_{3}(n)>0$ eventually. From (4), since the sequence $\left\{x_{3}(n)\right\}$ is eventually positive, the sequence $\left\{x_{2}(n)\right\}$ is eventually nondecreasing. Thus, $\lim _{n \rightarrow \infty} x_{2}(n)>0$. By Theorem 2 again, we get the assertion.

Theorem 4 If conditions (7)-(9) and (14) are satisfied, every eventually non-oscillatory solution $X=X(n), n \in \mathbb{Z}_{n_{0}-\eta}^{\infty}$ of (1) fulfilling condition (III) is bounded.

Proof Let $X=X(n), n \in \mathbb{Z}_{n_{0}-\eta}^{\infty}$ be an eventually non-oscillatory solution of (1). Without loss of generality, assume that $\left\{x_{1}(n)\right\}$ is eventually positive. By Lemma 4 , the sequences $\{z(n)\}$ and $\left\{x_{1}(n)\right\}$ are eventually monotonic. From (3), we deduce that $\{z(n)\}$ is nonincreasing. If $\{z(n)\}$ is bounded, then, by Lemma $2,\left\{x_{1}(n)\right\}$ is bounded as well and, therefore, there exists a finite $\lim _{n \rightarrow \infty} x_{1}(n)$. In that case, by Lemma 5 , we obtain the assertion. If $\{z(n)\}$ is unbounded, then, by Remark $2,\left\{x_{1}(n)\right\}$ is unbounded. The sequence $\left\{x_{1}(n)\right\}$ is nondecreasing and $\lim _{n \rightarrow \infty} x_{1}(n)=+\infty$. Therefore,

$$
\lim _{n \rightarrow \infty} z(n)=-\infty, \quad \lim _{n \rightarrow \infty} x_{1}(n)=+\infty
$$

This is a contradiction with (12) since

$$
z(n)=x_{1}(n)+p(n) x_{1}(n-\delta) \geq x_{1}(n-\delta)+p(n) x_{1}(n-\delta)=(1+p(n)) x_{1}(n-\delta)
$$

and

$$
\lim _{n \rightarrow \infty} z(n)=\lim _{n \rightarrow \infty}\left(x_{1}(n)+p(n) x_{1}(n-\delta)\right) \geq \lim _{n \rightarrow \infty}(1+p(n)) x_{1}(n-\delta)=+\infty
$$

\section{Examples}

The following examples illustrate Theorem 3 and Theorem 4. Among others, the examples demonstrate that the sets of solutions (I)-(III) defined in Theorem 1 are nonempty.

Example 1 Let $n_{0}>2$. In system (1), we set

$$
\begin{aligned}
& p(n)=\frac{1}{2}-\frac{1}{n}, \quad a_{1}(n)=\frac{3}{3 n+2}, \quad a_{2}(n)=\frac{1}{n+2} \\
& a_{3}(n)=\frac{1}{\sqrt[3]{n+1}\left(\sqrt[3]{(n+1)^{2}}+\sqrt[3]{(n+1)(n+2)}+\sqrt[3]{(n+2)^{2}}\right)} \\
& a_{4}(n)=\frac{\sqrt[3]{n+3}-\sqrt[3]{n+2}}{(n+1)^{5}+1}
\end{aligned}
$$


Moreover, we set

$$
\begin{array}{ll}
f_{1}(t)=\frac{3}{2} t+\operatorname{sgn} t, \quad f_{2}(t)=t^{3}+2 \operatorname{sgn} t, \quad f_{3}(t)=t, \\
f_{4}(t)=t^{5}+\operatorname{sgn} t, \quad \delta=2, \quad \tau=1 .
\end{array}
$$

All assumptions of Theorem 3 are satisfied. The solution $X=X(n)$ with

$$
x_{1}(n)=n+2, \quad x_{2}(n)=n+1, \quad x_{3}(n)=\sqrt[3]{n+1}, \quad x_{4}(n)=\sqrt[3]{n+2}
$$

is one of the unbounded solutions of system (1). It fulfills condition (I), defined in Theorem 1 .

Example 2 Let $n_{0}>2$. In system (1), we take

$$
\begin{aligned}
& p(n)=-\frac{1}{\sqrt{n}}, \\
& a_{1}(n)=\frac{1}{(\sqrt{n-2}+1)(\sqrt{n+2}+\sqrt{n+1})} \\
& a_{2}(n)=\frac{\sqrt{(n-2)^{3}}}{\left(1+\sqrt{(n-2)^{3}}\right)(\sqrt{n+1}+\sqrt{n})} \\
& a_{3}(n)=\frac{1}{\sqrt{n}(\sqrt{n+1}+\sqrt{n})}, \\
& a_{4}(n)=\frac{1}{\left(2+\sqrt{(n-1)^{3}}\right)(\sqrt{n+3}+\sqrt{n+4}) \sqrt{(n+3)(n+4)}}
\end{aligned}
$$

and

$$
\begin{aligned}
& f_{1}(t)=t+\operatorname{sgn} t, \quad f_{2}(t)=t^{3}+\operatorname{sgn} t, \quad f_{3}(t)=t, \\
& f_{4}(t)=t^{3}+2 \operatorname{sgn} t, \quad \delta=1, \quad \tau=2 .
\end{aligned}
$$

All assumptions of Theorem 3 are satisfied. The solution $X=X(n)$ with

$$
x_{1}(n)=\sqrt{n+1}, \quad x_{2}(n)=\sqrt{n}, \quad x_{3}(n)=\frac{1}{\sqrt{n}}, \quad x_{4}(n)=\frac{-1}{\sqrt{n+3}}
$$

is one of the unbounded solutions of system (1). This solution fulfills condition (II), defined in Theorem 1.

Example 3 We put $n_{0}>2$,

$$
p(n)=\frac{1}{25}, \quad a_{1}(n)=4, \quad a_{2}(n)=\frac{8}{5}, \quad a_{3}(n)=2, \quad a_{4}(n)=\frac{8}{125}
$$

and

$$
f_{1}(t)=f_{2}(t)=f_{3}(t)=f_{4}(t)=t, \quad \delta=2, \quad \tau=0
$$


in system (1). All assumptions of Theorem 4 are satisfied. The solution $X=X(n)$ with

$$
x_{1}(n)=\frac{1}{5^{n}}, \quad x_{2}(n)=\frac{-2}{5^{n+1}}, \quad x_{3}(n)=\frac{1}{5^{n+1}}, \quad x_{4}(n)=\frac{-2}{5^{n+2}},
$$

is one of the bounded solutions of system (1). This solution belongs to the class (III), defined in Theorem 1.

Competing interests

The authors declare that they have no competing interests.

\section{Authors' contributions}

The authors have made equivalent contributions. All authors read and approved the final manuscript.

\section{Author details}

${ }^{1}$ Brno University of Technology, Brno, Czech Republic. ${ }^{2}$ University of Białystok, Białystok, Poland. ${ }^{3}$ University of Žilina, Žilina, Slovakia.

\section{Acknowledgements}

The first author has been supported by the project No. LO1408, AdMaS UP-Advanced Materials, Structures and Technologies, (supported by Ministry of Education, Youth and Sports of the Czech Republic under the National Sustainability Programme I). The third author was supported by the project KEGA 004ŽU-4/2014 of the Cultural and Educational Grant Agency of the Ministry of Education, Science, Research and Sport of the Slovak Republic.

Received: 2 June 2015 Accepted: 7 October 2015 Published online: 15 October 2015

\section{References}

1. Došlá, Z, Krejčová, J: Oscillation of a class of the fourth-order nonlinear difference equations. Adv. Differ. Equ. (2012). doi:10.1186/1687-1847-2012-99

2. Došlá, Z, Krejčová, J: Asymptotic and oscillatory properties of the fourth-order nonlinear difference equations. Appl. Math. Comput. 249, 164-173 (2014)

3. Agarwal, RP, Grace, SR, Manojlović, JV: On the oscillatory properties of certain fourth order nonlinear difference equations. J. Math. Anal. Appl. 322, 930-956 (2006)

4. Agarwal, RP, Manojlović, JV: Asymptotic behavior of nonoscillatory solutions of fourth order nonlinear difference equations. Dyn. Contin. Discrete Impuls. Syst., Ser. A Math. Anal. 16, 155-174 (2009)

5. Migda, M, Musielak, A, Schmeidel, E: On a class of fourth order nonlinear difference equations. Adv. Differ. Equ. 2004(1), 23-36 (2004)

6. Migda, M, Musielak, A, Schmeidel, E: Oscillatory of fourth order nonlinear difference equations with quasidifferences. Opusc. Math. 26(2), 371-380 (2006)

7. Migda, M, Schmeidel, E: Asymptotic properties of fourth order nonlinear difference equations. Math. Comput. Model. 39, 1203-1211 (2004)

8. Popenda, J, Schmeidel, E: On the solution of fourth order difference equations. Rocky Mt. J. Math. 25(4), 1485-1499 (1995)

9. Schmeidel, E: Oscillation and nonoscillation theorems for fourth order difference equations. Rocky Mt. J. Math. 33(3), 1083-1094 (2003)

10. Schmeidel, E: Nonoscillation and oscillation properties for fourth order nonlinear difference equations. In: Aulbach, B, Elaydi, S, Ladas, G (eds.) New Progress in Difference Equations, pp. 531-538. CRC Press, Boca Raton (2004)

11. Schmeidel, E, Szmanda, B: Oscillatory and asymptotic behavior of certain difference equation. Nonlinear Anal. 47, 4731-4742 (2001)

12. Smith, B, Taylor, WE: Oscillatory and asymptotic behavior of certain fourth order difference equations. Rocky Mt. J. Math. 16(2), 403-406 (1986)

13. Graef, JR, Thandapani, E: Oscillatory and asymptotic behavior of fourth order nonlinear delay difference equations. Fasc. Math. 31, 23-36 (2001)

14. Thandapani, E, Arockiasamy, IM: On fourth order nonlinear oscillations of difference equations. Comput. Math. Appl. 42, 357-368 (2001)

15. Thandapani, E, Graef, JR: Oscillatory and asymptotic behavior of fourth order nonlinear delay difference equations. Fasc. Math. 31, 23-36 (2001)

16. Thandapani, E, Pandian, S, Dhanasekaran, R, Graef, J: Asymptotic results for a class of fourth order quasilinear difference equations. J. Differ. Equ. Appl. 13(12), 1085-1103 (2007)

17. Thandapani, E, Selvaraj, B: Oscillatory and nonoscillatory behavior of fourth order quasilinear difference equations. Far East J. Appl. Math. 17, 287-307 (2004)

18. Thandapani, E, Vijaya, M: Oscillatory and asymptotic behavior of fourth order quasilinear difference equations. Electron. J. Qual. Theory Differ. Equ. 2009, 64 (2009)

19. Yan, J, Liu, B: Oscillatory and asymptotic behavior of fourth order difference equations. Acta Math. Sin. 13, 105-115 (1997)

20. Diblík, J, Hlavičková, I: Combination of Liapunov and retract methods in the investigation of the asymptotic behavior of solutions of systems of discrete equations. Dyn. Syst. Appl. 18(3-4), 507-537 (2009)

21. Stevič, S, Diblík, J, Iričanin, B, Šmarda, Z: On a third-order system of difference equations with variable coefficients. Abstr. Appl. Anal. 2012, Article ID 50852 (2012) 
22. Stevič, S, Diblík, J, Iričanin, B, Šmarda, Z: Solvability of nonlinear difference equations of the fourth order. Electron. J. Differ. Equ. 2014, 264 (2014)

23. Schmeidel, E: Boundedness of solutions of nonlinear three-dimensional difference systems with delays. Fasc. Math. 44, 107-113 (2010)

24. Schmeidel, E: Oscillation of nonlinear three-dimensional difference systems with delays. Math. Bohem. 135(2), 163-170 (2010)

25. Schmeidel, E: Asymptotic trichotomy for positive solutions of a class of fourth order nonlinear neutral difference equations with quasidifferences. Nonlinear Anal. 63(5-7), e899-e907 (2005)

26. Schmeidel, E, Schmeidel, J: Asymptotic behavior of solutions of a class of fourth order nonlinear neutral difference equations with quasidifferences. Tatra Mt. Math. Publ. 38, 243-254 (2007)

27. Migda, M, Migda, J: Asymptotic properties of solutions of second-order neutral difference equations. Nonlinear Anal. 63, e789-e799 (2005)

28. Jankowski, R, Schmeidel, E, Zonenberg, J: Oscillatory properties of solutions of the fourth order difference equations with quasidifferences. Opusc. Math. 34(4), 789-797 (2014)

\section{Submit your manuscript to a SpringerOpen ${ }^{\circ}$ journal and benefit from:}

- Convenient online submission

- Rigorous peer review

- Immediate publication on acceptance

- Open access: articles freely available online

- High visibility within the field

- Retaining the copyright to your article 